\title{
LGI1 antibody encephalopathy overlapping with sporadic Creutzfeldt-Jakob disease
}

\section{OPEN}

Boaz Kim, BMedSci*

Patrick Yoo, MSc*

Tom Sutherland, MBBS, MMed

Alison Boyd, DipAppSci

Christiane Stehmann,

$\mathrm{PhD}$

Catriona McLean, MBBS, MD

Steven Collins, MBBS,

MD

Correspondence to

Dr. Collins:

stevenjc@unimelb.edu.au

\section{ABSTRACT}

Objective: To report a rare case of leucine-rich, glioma inactivated 1 (LGI1) antibody-mediated autoimmune encephalopathy clinically overlapping with pathologically confirmed sporadic CreutzfeldtJakob disease (CJD).

Methods: The patient was investigated with repeated brain MRI, EEG, CSF examination, wholebody fluorodeoxy-glucose positron emission tomography, genetic analysis of the prion protein gene (PRNP), and extensive serologic screening for paraneoplastic and autoimmune encephalopathy markers. Written informed consent was obtained from the patient's next of kin for access to clinical files for research purposes and for publication.

Results: The patient was a 77-year-old man who presented with faciobrachial dystonic seizures (FBDS) secondary to LGI1 antibody-mediated autoimmune encephalopathy, with suggestive MRI findings and a complete response to treatment with combinatorial immunosuppression. Stereotactic biopsy of a nonenhancing T1 hyperintense basal ganglia lesion during the initial FBDS phase, albeit following immunosuppression, did not disclose evidence of lymphocytic inflammation. Following full remission of the FBDS, the patient manifested a rapidly progressive dementia associated with gross motor decline confirmed to be CJD at autopsy (molecular subtype VV3), with no evidence of a pathogenic PRNP mutation.

Conclusions: Our patient highlights that these rare diseases are not invariably mutually exclusive and underscores the benefits of comprehensive neuropathologic examination of the brain to achieve an accurate diagnosis, especially in complex cases when the clinical trajectory dramatically deviates and a concomitant disease may need to be conscientiously considered to best explain the new clinical course. Neurol Neuroimmunol Neuroinflamm 2016;3:e248; doi: 10.1212/ NXI.0000000000000248

\section{GLOSSARY}

BG = basal ganglia; CJD = Creutzfeldt-Jakob disease; FBDS = faciobrachial dystonic seizures; $\mathbf{s C J D}=$ sporadic Creutzfeldt-Jakob disease; VGKC = voltage-gated potassium channel; VGKCC-LE = voltage-gated potassium channel complex limbic encephalitis.

A patient presented at age 77 years with a 1-week history of intermittent episodes of left-sided spasms involving the arm, face, shoulder, or leg, lasting 4-5 seconds, which were accompanied by recurrent falls but not associated with loss of consciousness. Medical history included type 2 diabetes mellitus managed with metformin and glimepiride; hypertension treated with atenolol and telmisartan; hypercholesterolemia controlled with simvastatin; and chronic obstructive pulmonary disease, for which salbutamol and tiotropium inhalant therapy were prescribed. There was no history of neurologic disorders. CT and MRI of the brain, as well as EEG, proved unremarkable. Neurologic examination results were normal and the patient had apparently normal cognitive

\footnotetext{
*These authors contributed equally to this work.

From the Australian National Creutzfeldt-Jakob Disease Registry, The Florey Institute of Neuroscience and Mental Health (B.K., P.Y., A.B., C.S., C.M., S.C.), and Department of Medicine, Royal Melbourne Hospital (A.B., S.C.), The University of Melbourne, Parkville; Melbourne Medical School (B.K., P.Y.), The University of Melbourne; Medical Imaging Department (T.S.), St. Vincent's Hospital Melbourne, Fitzroy; and Department of Anatomical Pathology (C.M.), Alfred Health, Prahran, Australia.

Funding information and disclosures are provided at the end of the article. Go to Neurology.org/nn for full disclosure forms. The Article Processing charge was paid by the authors.

This is an open access article distributed under the terms of the Creative Commons Attribution-NonCommercial-NoDerivatives License 4.0 (CC BY-NC-ND), which permits downloading and sharing the work provided it is properly cited. The work cannot be changed in any way or used commercially.
} 
function by informal assessment. The patient was commenced on phenytoin (300 mg daily) following a provisional diagnosis of partial epilepsy.

Approximately 1 month later, the patient was admitted to the hospital because of recurrence of the left facial and upper limb spasms of increased frequency, sometimes occurring every 8-10 minutes, with up to 30 episodes daily; minor difficulties with cognition were also apparent. During the admission, repeat MRI of the brain (figure 1A) showed T1 hyperintensity in the right basal ganglia $(B G)$, which was nonenhancing and appeared associated with modest mass effect. Based on the new imaging findings and concerns about neoplastic infiltration, anticonvulsant therapy was changed to carbamazepine $200 \mathrm{mg}$ twice daily and empirical treatment with oral dexamethasone was commenced. Shortly thereafter, the patient underwent stereotactic biopsy of the T1 hyperintense lesion, which only revealed mild, nonspecific, astrocytic gliosis but no evidence of neoplasia or inflammation. Routine investigations were unremarkable aside from a new-onset hyponatremia (122 mM; normal 135-145 mM). Investigations for an underlying neoplastic process included CT imaging of the chest-abdomen-pelvis, whole body fluorodeoxy-glucose positron emission tomography with CT correlation (FDG-PET/CT), and serum paraneoplastic antibody and tumor marker screening (including anti-Hu and anti-Yo/Purkinje cell antibodies and carcinoembryonic antigen CA 19-9), which were negative. CSF was normal and prolonged scalp EEG monitoring during episodes of spasms showed no evidence of focal epileptiform activity. An autoimmune encephalopathy screen for anti-NMDA receptor antibodies in both serum and CSF was negative but anti-voltage-gated potassium channel (VGKC) complex antibodies were positive by radioimmunoassay at $338 \mathrm{pM}$ (normal <85). Posthumous retesting of the patient's serum using indirect immunofluorescence (Euroimmun, Lübeck, Germany)

Figure 1 Prebiopsy and postbiopsy MRI

T1 without contrast

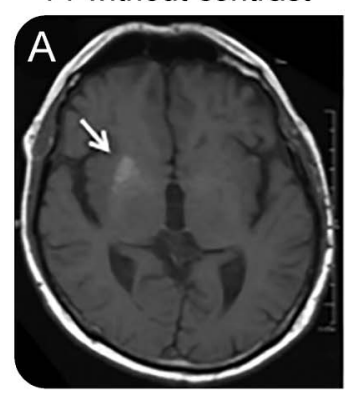

T1 without contrast

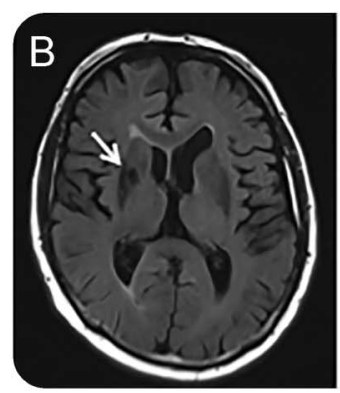

T1 with contrast

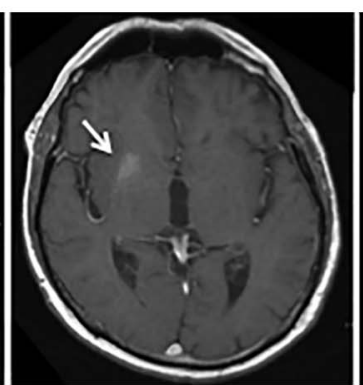

FLAIR

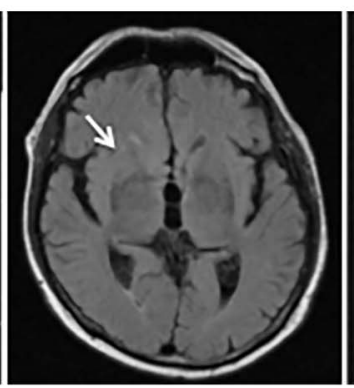

DWI

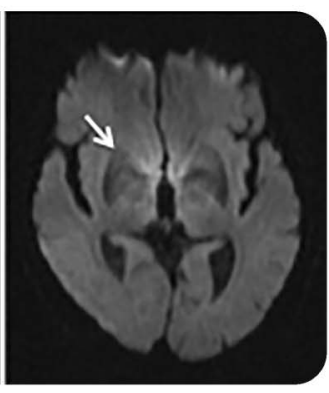

FLAIR

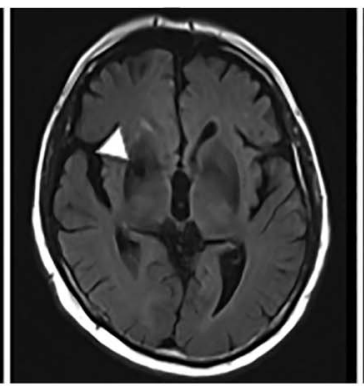

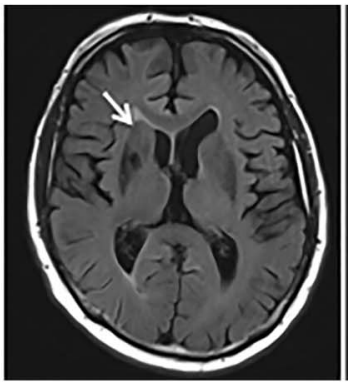

DWI

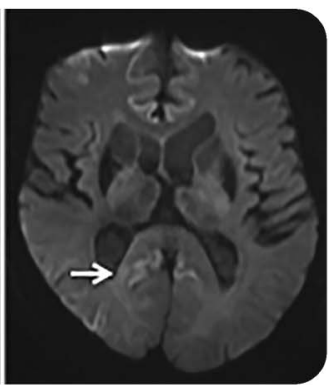

(A) Immediately prebiopsy, axial brain MRI shows a non-contrast enhancing T1 hyperintensity in the right basal ganglia juxtaposed to the external capsule (arrows) with modest mass effect; these changes were not evident in a prior MRI study. Corresponding fluid-attenuated inversion recovery (FLAIR) and diffusion-weighted imaging (DWI) sequences were essentially unremarkable. (B) Postbiopsy axial brain MRI reveals resolution of the right basal ganglia T1 hyperintensity (arrow), with biopsy artefact (arrow head), with corresponding FLAIR images remaining otherwise unremarkable aside from ongoing modest mass effect (arrow), while the DWI series displays subtle cerebral cortical signal increase in the posterior cingulate gyri (right more than left) and right posterior temporal region (arrow). 
confirmed the presence of antibodies to LGI1 but not to contactin-associated protein-like 2 . Based on all the available clinical information at the time of the anti-VGKC antibody result, the working diagnosis of faciobrachial dystonic seizures (FBDS) secondary to antiVGKC encephalopathy was made. The patient was changed from dexamethasone (after approximately 1 month of treatment) to prednisolone (25 mg daily) and azathioprine (100 mg daily), with monthly IV immunoglobulin given for 4 months as combinational immunotherapy, which resulted in complete clinical remission of his FBDS and partial improvement in cognition, allowing the patient to be discharged home with gradual outpatient tapering of maintenance corticosteroid therapy.

Approximately 4 months after discharge, despite good ongoing control of the FBDS, the patient's cognition began to decline again (Mini-Mental State Examination 19/30), with minimal response to escalation of the prednisolone to $25 \mathrm{mg}$ daily. Cognitive impairment at that time included amnesia, executive dysfunction, visuospatial impairment, and poor insight. Repeat brain MRI confirmed absence of the previous right $\mathrm{BG}$ T1 hyperintensity (with postbiopsy changes evident) and equivocal cerebral cortical signal abnormality restricted to the posterior cingulate gyri (right more than left) and posterior right temporal lobe on the diffusion-weighted series (figure 1B). Repeat EEG showed only nonspecific generalized slowing consistent with a moderate encephalopathy. The patient thereafter manifested inexorable cognitive and gross motor decline, associated with bulbar dysfunction and double incontinence, dying 6 months later, spending the final 2 months of his life in high-level care. The total illness duration from clinical presentation to death was 17 months, the first 8 months predominantly involving autoimmune FBDS and its management and the next 9 months related to a rapidly progressing dementia with profound functional decline.

A diagnosis of Creutzfeldt-Jakob disease (CJD) was confirmed at autopsy, characterized by widespread spongiform encephalopathy associated with neuronal loss and astrocytic gliosis (figure 2). There was no evidence of any lymphocytic inflammatory reaction. Genetic studies did not reveal any pathogenic variants of the prion protein gene (PRNP) and the patient was homozygous valine/valine (VV) at codon 129. Western blot showed evidence of proteaseresistant prion protein, classified as type 3 according to the nomenclature of Collinge et al. ${ }^{1}$ As follow-up to the brain pathologic findings at autopsy, formal re-review of the stereotactic biopsy was undertaken but did not reveal previously unappreciated spongiform changes and immunohistochemistry for abnormal prion protein deposits was negative.

DISCUSSION We propose that the 17-month illness to the time of death in our patient most likely comprised 2 overlapping neurologic illnesses: anti-LGI1 autoantibody-mediated encephalopathy predominantly

Figure 2 Neuropathologic diagnosis of Creutzfeldt-Jakob disease (CJD) at postmortem

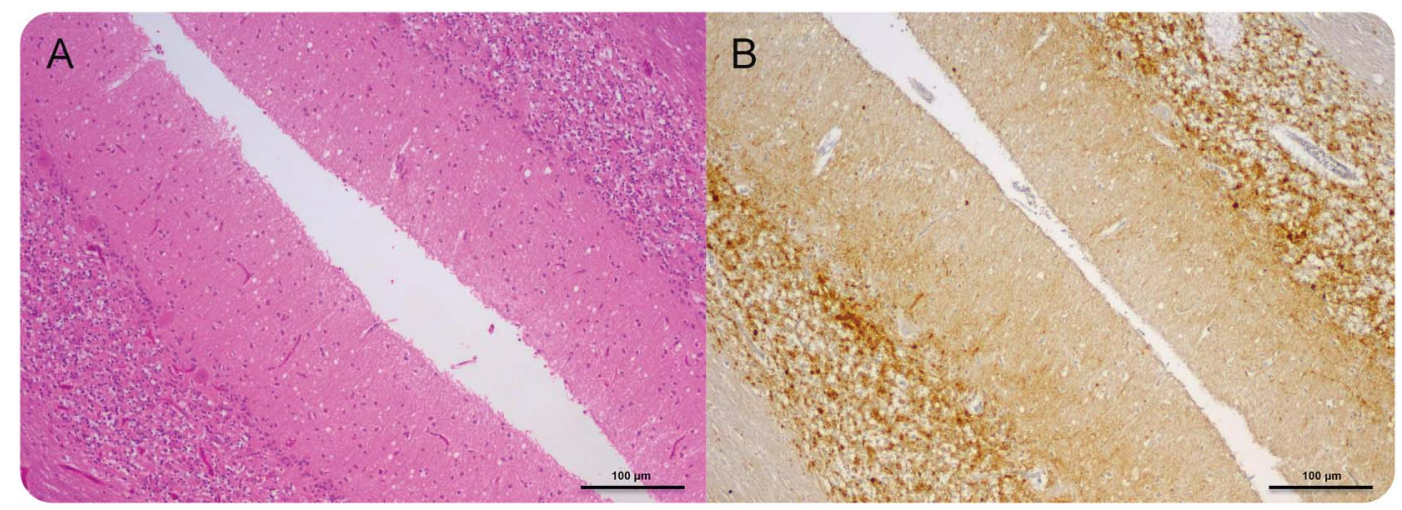

(A) Cerebellar vermis shows typical changes of CJD including spongiform encephalopathy, gliosis, and neuronal loss (hematoxylin and eosin $\times 100$ actual magnification) and (B) synaptic pattern (PrP) immunoreactivity (12 F10 PrP antibody $\times 100$ actual magnification). Note there is no evidence of any inflammatory cells. 
manifesting as FBDS and inexorable cognitive and gross motor decline due to VV3 sporadic CJD (sCJD). While the FBDS was completely responsive to immunosuppression, the patient's early cognitive impairment was only partially responsive, and although recognized for LGI1 antibody encephalopathy raises the possibility that at least some of this impairment may have related to sCJD before more dramatic cognitive decline and accompanying features were evinced. Although we consider it unlikely, due to the response of the FBDS to immunosuppression in the setting of anti-LGI1 antibodies and typical MRI changes, we cannot exclude the possibility that the patient's illness reflected an atypical CJD phenotype from the time of first presentation with FBDS.

FBDS is a recently described immunotherapyresponsive seizure disorder characterized by highly stereotyped clinical features and the presence of autoantibodies to LGI1, a protein component of the VGKC complex. ${ }^{2-4}$ Furthermore, FBDS is considered to be a highly specific phenotype and almost pathognomonic for LGI1 autoantibody-mediated disease. ${ }^{5,6}$ FBDS have also been recognized as a frequent prodromal sign preceding LGI1 antibody limbic encephalitis, ${ }^{5}$ a neuroinflammatory condition characterized by a clinical triad of FBDS, cognitive impairment, and hyponatraemia. ${ }^{6}$ The absence of cognitive deficits at presentation with FBDS renders it more likely that our patient was in the prodromal phase prior to the onset of limbic encephalitis, which is consistent with the absence of T2/fluid-attenuated inversion recovery signal increase in the mesiotemporal lobes typically seen in limbic encephalitis. ${ }^{7}$ In the early stage of the illness, MRI demonstrated a nonenhancing T1 hyperintensity in the right BG, typical of what is observed in a sizeable proportion of patients presenting with FBDS secondary to anti-LGI1 antibody encephalopathy. In a recent retrospective case series of 26 patients with FBDS and positive serology for LGI1 autoantibodies, 10 of these patients had the novel finding of contralateral BG T1 hyperintensity, thereby providing evidence for BG T1 hyperintensity as a useful MRI biomarker of anti-LGI1 autoimmune FBDS. ${ }^{2}$ Our patient also developed hyponatremia (Na $122 \mathrm{mM}$ ), a common finding in FBDS and limbic encephalitis. ${ }^{5-7}$

LGI1 antibody-mediated FBDS and limbic encephalitis have been reported to show variable response rates to immunotherapy, with most cases reporting at least partial improvement of FBDS and cognitive symptoms. Moreover, early initiation of immunotherapy at the FBDS stage can prevent progression to limbic encephalitis. ${ }^{8}$ Our patient demonstrated an initial good response of the FBDS to combination immunotherapy and anticonvulsant medication, with partial improvement in cognition, before eventually manifesting inexorable intellectual, behavioral, and gross motor decline almost certainly due to co-occurrence of sCJD.

Occasional diagnostic confusion between CJD and autoimmune encephalopathies is well-recognized. Although positive VGKC complex (and in retrospect LGI1) antibodies were present at the early stage of our patient's illness, there have been reports of elevated VGKC complex antibody levels in patients with CJD, albeit with no symptoms of FBDS or other evidence of autoimmune encephalopathy, with subsequent uncertainty about the significance of antibody levels in these clinical situations. ${ }^{9}$ Further illustrating the diagnostic challenges that can arise in the evaluation of patients with neurologic illnesses with positive serology for autoimmune encephalopathies, previous case series have discussed autoimmune VGKC complex limbic encephalitis (VGKCC-LE) as a mimic of CJD, ${ }^{10}$ with patients initially thought to have CJD but later confirmed to have immunotherapy-responsive VGKCCLE, and conversely, CJD has been reported as a mimic of VGKCC-LE ${ }^{11}$ and of Morvan syndrome associated with elevated VGKCC and glycine receptor autoantibodies. ${ }^{12}$ Our patient was somewhat different from the usual clinical reports, manifesting a dramatic biphasic illness punctuated by a period of incomplete recovery, rather than a predominantly monophasic course, until an accurate diagnosis was achieved. Development of refractory cognitive and gross motor decline in our patient prompted some uncertainty and reconsideration of the diagnosis but was construed as probably reflecting treatment-unresponsive LGI1 antibody encephalopathy rather than representing the development of a second neurologic illness such as sCJD, especially when there was no definite objective investigational evidence to clearly support such an alternative diagnosis.

The absence of inflammation in the brain at biopsy and autopsy examinations is without definite explanation but we conjecture most likely represents the effects of immunosuppressant treatment in a patient who appeared to have at least a partially responsive anti-LGI1 antibody encephalopathy. Recent reports of anti-LGI1 antibody encephalopathy are largely devoid of neuropathologic findings. $2,3,5,6,11$

Our patient underscores the benefits of comprehensive neuropathologic examination of the brain to achieve an accurate diagnosis, especially in complex cases when the clinical trajectory dramatically deviates and vigilance for a concomitant disease is arguably the best approach to explain the new clinical course. In addition, our patient exemplifies the utility of recognizing BG T1 hyperintensity in patients with brief adventitious face and limb movements suggestive of FBDS, which should prompt investigation for VGKC complex antibodies and in particular anti-LGI1 antibodies, which may be responsive to immunosuppressive therapy. 


\section{AUTHOR CONTRIBUTIONS}

B. Kim: involved in the collection of patient information and creation of figure 1 and contributed to the manuscript writing, drafting, and reviewing process. P. Yoo: involved in the collection of patient information and creation of figure 1 and contributed to the manuscript writing, drafting, and reviewing process. T. Sutherland: reviewed clinical images, assisted in the creation of figure 1 , and contributed to the drafting and review of the manuscript. A. Boyd: assisted in collection of patient clinical files and in the manuscript drafting and review process. C. Stehmann: assisted in collection of patient clinical files and in the manuscript drafting and review process. C. McLean: reviewed the pathologic details of the case and contributed to the drafting and review process and creation of figure 2. S. Collins: provided overall guidance in the direction of the case report and contributed to the writing, drafting, and reviewing process.

\section{ACKNOWLEDGMENT}

The ANCJDR thanks the patient's family and associated health care personnel for their support in the preparation of the case report.

\section{STUDY FUNDING}

The Australian National Creutzfeldt-Jakob Disease Registry (ANCJDR) is funded by the Commonwealth Department of Health. S.C. was funded in part by NHMRC Practitioner Fellowship (APP1005816).

\section{DISCLOSURE}

B.S. Kim and P. Yoo report no disclosures. T. Sutherland is on the editorial board for Insights into Imaging. A. Boyd received research support from Australian Commonwealth Department of Health. C. Stehmann holds a patent for albumin depletion technology. C. McLean reports no disclosures. S. Collins is an associate editor for Journal of Alzheimer Disease and received research support from NHMRC, Rebecca Cooper Foundation, Brain Foundation, and Commonwealth Department of Health. Go to Neurology.org/nn for full disclosure forms.

Received February 25, 2016. Accepted in final form April 7, 2016.

\section{REFERENCES}

1. Collinge J, Sidle KC, Meads J, Ironside J, Hill AF. Molecular analysis of prion strain variation and the aetiology of “new variant” CJD. Nature 1996;383:685-690.
2. Flanagan EP, Kotsenas AL, Britton JW, et al. Basal ganglia T1 hyperintensity in LGI1-autoantibody faciobrachial dystonic seizures. Neurol Neuroimmunol Neuroinflamm 2015;2:e161. doi: 10.1212/NXI.0000000000000161.

3. Lai M, Huijbers MGM, Lancaster E, et al. Investigation of LGI1 as the antigen in limbic encephalitis previously attributed to potassium channels: a case series. Lancet Neurol 2010;9:776-785.

4. Irani SR, Buckley C, Vincent A, et al. Immunotherapyresponsive seizure-like episodes with potassium channel antibodies. Neurology 2008;71:1647-1648.

5. Irani SR, Michell AW, Lang B, et al. Faciobrachial dystonic seizures precede Lgil antibody limbic encephalitis. Ann Neurol 2011;69:892-900.

6. Casault C, Alikhani K, Pillay N, Koch M. Jerking \& confused: leucine-rich glioma inactivated 1 receptor encephalitis. J Neuroimmunology 2015;289:84-86.

7. Bien CG, Elger CE. Limbic encephalitis: a cause of temporal lobe epilepsy with onset in adult life. Epilepsy Behav 2007;10:529-538.

8. Irani SR, Stagg CJ, Schott JM, et al. Faciobrachial dystonic seizures: the influence of immunotherapy on seizure control and prevention of cognitive impairment in a broadening phenotype. Brain 2013;136:3151-3162.

9. Jammoul A, Lederman RJ, Tavee J, Li Y. Presence of voltage-gated potassium channel complex antibody in a case of genetic prion disease. BMJ Case Rep 2014; 2014.

10. Geschwind MD, Tan KM, Lennon VA, et al. Voltage-gated potassium channel autoimmunity mimicking CreutzfeldtJakob disease. Arch Neurol 2008;65:1341-1346.

11. Newey CR, Appleby BS, Shook S, Sarwal A. Patient with voltage-gated potassium-channel (VGKC) limbic encephalitis found to have Creutzfeldt-Jakob disease (CJD) at autopsy. J Neuropsychiatry Clin Neurosci 2013;25:E05-E07.

12. Angus-Leppan H, Rudge P, Mead S, Collinge J, Vincent A. Autoantibodies in sporadic Creutzfeldt-Jakob disease. JAMA Neurology 2013;70:919-922. 


\section{Neurology \\ Neuroimmunology \& Neuroinflammation}

\section{LGI1 antibody encephalopathy overlapping with sporadic Creutzfeldt-Jakob disease Boaz Kim, Patrick Yoo, Tom Sutherland, et al. \\ Neurol Neuroimmunol Neuroinflamm 2016;3; \\ DOI 10.1212/NXI.0000000000000248}

This information is current as of June 16, 2016

\section{Updated Information \& Services}

References

Subspecialty Collections

Permissions \& Licensing

Reprints including high resolution figures, can be found at:

http://nn.neurology.org/content/3/4/e248.full.html

This article cites 11 articles, 0 of which you can access for free at: http://nn.neurology.org/content/3/4/e248.full.html\#\#ref-list-1

This article, along with others on similar topics, appears in the following collection(s):

All Clinical Neurology

http://nn.neurology.org//cgi/collection/all_clinical_neurology

Autoimmune diseases

http://nn.neurology.org//cgi/collection/autoimmune_diseases

MRI

http://nn.neurology.org//cgi/collection/mri

Prion disease; see Infections/prion

http://nn.neurology.org//cgi/collection/prion_disease

Information about reproducing this article in parts (figures,tables) or in its entirety can be found online at:

http://nn.neurology.org/misc/about.xhtml\#permissions

Information about ordering reprints can be found online:

http://nn.neurology.org/misc/addir.xhtml\#reprintsus

Neurol Neuroimmunol Neuroinflamm is an official journal of the American Academy of Neurology.

Published since April 2014, it is an open-access, online-only, continuous publication journal. Copyright $@$ 2016 American Academy of Neurology. All rights reserved. Online ISSN: 2332-7812.

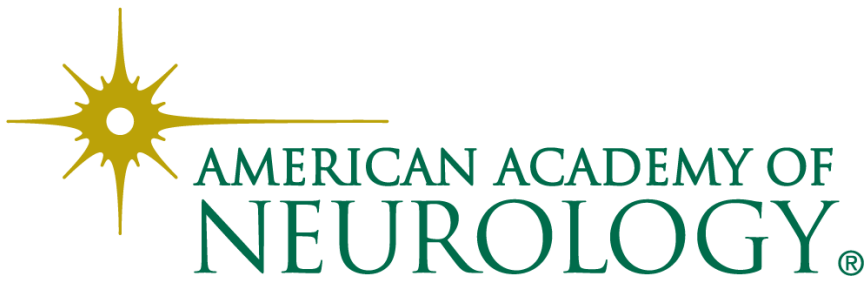

